\title{
CALCULATION OF PRESCRIBED DAILY DOSE OF ANTICOAGULANTS IN SOUTH INDIAN POPULATION
}

\author{
VENKATESHWARLU K, JOSHUA JACINTH TP, PRANEETH G, ANUSHA G, RAHUL GOUD N
}

Department of Pharmacy Practice, CMR College of Pharmacy, Hyderabad, Telangana, India. Email: venkipharmd@gmail.com

Received: 01 December 2017, Revised and Accepted: 22 February 2018

\section{ABSTRACT}

Objectives: The objectives of this study were to identify the commonly prescribed anticoagulants and to study the prescription pattern of anticoagulants in various indications and to calculate prescribing daily dose (PDD) and to compare PDD with defined daily dose of anticoagulants.

Methods: A total of 119 patients of all age groups, either of sex who are receiving anticoagulant therapy were included in this prospective observational study done in limited period of 6 months in the inpatient department of general medicine, gynecology, pediatrics, and surgery. Patients who are noncooperative, cancer patients, psychiatric patients, and patients on hemodialysis and receiving anticoagulant treatment, and patients suffering from end-stage renal disease and hepatic failure are excluded from the study.

Results: In this study, we found that heparin, Low-molecular-weight heparins (LMWHs), warfarin, and acenocoumarol are the most commonly used drugs, of which heparin is highly prescribed. The use of anticoagulants in cardiovascular diseases was found to be high compared to other indications. PDD: DDD of warfarin is 0.46 , acenocoumarol is 0.75 , i.e., half the dose of drug showed its effectiveness, whereas heparin is 1.86 , LMWH's is 2.9 , i.e., double the dose is used to treat the disease effectively. A total of eight adverse drug reactions are observed with Vitamin K antagonists, i.e., $6.72 \%$.

Conclusion: We observed that the anticoagulants were not prescribed as the WHO defined doses in the study site. Vitamin K antagonists were prescribed at half of the WHO defined doses and heparin derivatives were prescribed at increased doses and sometimes double the WHO defined doses; these variations may be due to disease characteristics or patient characteristics or drug characteristics.

Keywords: Drug utilization evaluation, Anticoagulants, Prescribing daily dose, Defined daily dose.

(C) 2018 The Authors. Published by Innovare Academic Sciences Pvt Ltd. This is an open access article under the CC BY license (http://creativecommons. org/licenses/by/4. 0/) DOI: http://dx.doi.org/10.22159/ajpcr.2018.v11i6.23467

\section{INTRODUCTION}

"Drug utilization review/evaluation (DUR/DUE) is defined as the marketing, distribution, prescription, and use of drugs in a society with special emphasis on the resulting medical, social, and economic consequences" [1]. It was defined by the WHO in 1977 [2]. It is a powerful tool to evaluate present patterns/fashion of drug use and appropriateness of prescriptions. It is a descriptive and analytical method of collection, quantification, understanding, and evaluation of the prescribing pattern, and also dispensing and consumption for the advancement of existing treatment and enhancement of patient safety. It provides information about pattern, quality, and outcome of drug use [3]. And to promote rational and appropriate use of drugs of narrow therapeutic index. The anatomical and therapeutic chemical classification systems are recommended by the WHO [2]. The system is also used by International Drug Monitoring Centre, Uppsala, for classifying adverse drug reactions (ADRs). Defined daily dose (DDD) is the average of the maintenance dose per day which is used as a comparable unit. Prescribed daily dose (PDD) is not always equal to DDD. They both are a rough estimate of drug utilization. The primary outcome of the study is to calculate the PDD and secondary outcome of the study is to compare the PDD with DDD (WHO DDD) and also to identify the commonly prescribed anticoagulants in various indications.

\section{PDD:DDD method of DUR}

For each and every drug, DDD is defined by the WHO collaborating center for drug statistics and methodology as the assumed average maintenance adult dose per day for its main indication. Therefore, DDD is an international unit serving for regional and international comparisons. DDD is assumed average maintenance dose per day for a drug which is used for its main indication only in adults. It is a unit of measurement and does not correspond to PDD. Individual dosage regimen will differ based on patient groups, age, and weight; hence, they differ from DDD [4].

PDD is defined as the average dose prescribed according to a representative sample of prescriptions. It is important to relate the PDD to the diagnosis on which the dosage is based. The PDD will give the average daily amount of a drug that is actually prescribed. When there is a substantial difference between the PDD and the DDD, it is important to take this into consideration when evaluating and interpreting drug utilization figures, particularly in terms of morbidity [5].

Anticoagulants have a wide spectrum of use and associated risk with their therapy due to their narrow therapeutic range. Deep vein thrombosis, pulmonary embolism, myocardial infarction, unstable angina, rheumatic heart disease, vascular surgery, and prosthetic heart valve are the conditions where anticoagulants are widely used $[5,6]$. Titrating doses within their narrow therapeutic ranges prove to be difficult and either overdosing or underdosing occur which leads potential amount of risks. Due to the complications that can arise from the use of anticoagulants, a drug utilization pattern study should be done on the routinely prescribed anticoagulants [7]. This helps in evaluating the effectiveness of anticoagulant therapy, enhancing the safety of anticoagulants, achieving better rationality and providing feedback to the prescribers, and other healthcare personnel. Anticoagulants are a class of drugs that prevent blood coagulation either by inhibiting action of clotting factors or interfere with synthesis of coagulation factors that is they are used to prevent thrombus extension and embolic complications by reducing rate of fibrin formation, but they do not dissolve already formed clot but prevent recurrences [8]. They are classified into coumarin derivatives (vitamin dose [PDD] in various conditions such as deep vein thrombosis, pulmonary embolism, 
myocardial infarction, stroke, and atrial fibrillation and compare prescribing daily dose [PDD] with DDD of anticoagulants and Vitamin K antagonists), indirect thrombin inhibitors, direct thrombin inhibitors, and direct factor Xa inhibitors [9]. The target of this study is to identify the commonly prescribed anticoagulants and calculate PDD and compare it with DDD [10].

\section{METHODOLOGY}

This is a prospective observational study conducted over a period of 1 year (March 2016-March 2017). All the patients those are admitted in hospital reviewed on daily bases. Those are reached my inclusion criteria such as receiving anticoagulant therapy, all age groups, either of sex. Patients who are non-cooperative, cancer patients, psychiatric patients, and patients on hemodialysis and receiving anticoagulant treatment, and patients suffering from endstage renal disease and hepatic impairment are excluded from the study. A total of 119 patients enrolled into the study and collected all necessary information such as patient demographic information age, sex, area, and past medical history by interviewing the patients and patients caretakers. Laboratory reports collected to identify the diagnosis as well as rolled out exclusion criteria from the case sheet also collected medication information such as active ingredient name, dose, frequency, and duration of taken by patient. All the information was collected in well-designed data collection form. We calculated PDD (=total dose divided by the number of days) and expressed them as the PDD:DDD ratio (= amount of DDD per day and person). The DDDs for the selected anticoagulants were taken from precalculated DDD from the WHO. Heparin and low-molecularweight heparin (LMWH) were expressed in TU (10,000 Units) so they have being converted to units for convenience of the study. The calculated PDD of the anticoagulants is compared with DDD using simple t-test. The DDDs of anticoagulants defined by the WHO are warfarin $7.5 \mathrm{mg}$, acenocoumarol $5 \mathrm{mg}$, heparin $10 \mathrm{TU}$, and LMWH 2 TU. All the collected data were entered into Microsoft Excel. The raw data were taken into SAS software using Proc Import and data were redesigned/reorganized with number and character function, and PDD was calculated using Proc means and compared PDD with DDD using Proc t-test (simple t-test). In Proc t-test, $\mathrm{H}_{0}$ was considered as DDD. The minimum level of statistical significance was considered as $\mathrm{p}=0.05$.

\section{RESULTS}

During the study period, 1500 patients were reviewed, out of which $119(7.93 \%)$ cases were enrolled into the study according to the inclusion criteria, the remaining 1381 (92.06\%) patients were excluded based on the exclusion criteria, 730 (52.86\%) patients were excluded because there is not prescribed with anticoagulant, 320 (23.17\%) patients hesitated to share their medication-related information, $186(13.46 \%)$ patients on hemodialysis, $121(8.76 \%)$ patients were suffering from end-stage renal disease, $15(1.08 \%)$ patients were diagnosed with cancer, and $9(0.65 \%)$ patients were suffering from psychiatric illness.

Of 119 patients enrolled into the study, $61(51.26 \%)$ patients were diagnosed with coronary artery disease (CAD), 23 (19.33\%) patients were diagnosed with cerebrovascular accident (CVA), 21 (18.4\%) patients were diagnosed with deep vein thrombosis (DVT), and $13(10.92 \%)$ were diagnosed with other diseases which includes atrial fibrillation, chronic rheumatic heart disease (CRHD), and left ventricular dysfunction.

CAD was found to be predominant accounting 61 (51.26\%) patients, CVA was recorded at $23(19.33 \%)$ patients, DVT patients were $21(18.4 \%)$, and other diseases accounted for $14(10.92 \%)$ patients out of $119(100 \%)$.

In 119 patients, a total of 148 anticoagulants were prescribed. In 61 CAD cases, 64 anticoagulants were prescribed, in 23 CVA, 45 anticoagulants were prescribed, in 21 cases, 25 anticoagulants were prescribed, and in 14 other cases, 14 anticoagulants were prescribed across other diseases (Table 1).

A total of 148 prescriptions heparin were prescribed 70 times, LMWH was prescribed 31 times, warfarin was prescribed 23 times, and acenocoumarol was prescribed 24 times (Table 2).

All the anticoagulants' PDD was calculated for various indications. PDD was calculated as such by taking total dose of the drug and dividing it by number of days (Table 3).

Table 1: Patients distributed according to diagnosis

\begin{tabular}{lll}
\hline S. No. & Disease & Number of patients (\%) \\
\hline 1. & CAD & $61(51.26)$ \\
2. & CVA & $23(19.33)$ \\
3. & DVT & $21(18.4)$ \\
4. & Others (AF, CRHD, LVD, etc.) & $14(10.92)$ \\
\hline
\end{tabular}

CAD: Coronary artery disease, CVA: Cerebrovascular accident, DVT: Deep vein thrombosis, AF: Atrial fibrillation, CRHD: Chronic rheumatic heart disease, LVD: Left ventricular dysfunction

Table 2: Anticoagulants identified for various indications

\begin{tabular}{llllll}
\hline S. No. & Diagnosis & $\mathbf{n}$ & Drug & $\begin{array}{l}\text { Number } \\
\text { of times } \\
\text { prescribed }\end{array}$ & \% \\
\hline 1. & CAD & \multirow{2}{*}{61} & Heparin & 46 & 67.2 \\
& & & LMWH & 8 & 13.1 \\
& & & Warfarin & 3 & 4.9 \\
& & & Acenocoumarol & 7 & 11.4 \\
2. & CVA & 23 & Heparin & 10 & 43.4 \\
& & & LMWH & 15 & 65.2 \\
& & & Warfarin & 14 & 60.8 \\
& & & Acenocoumarol & 6 & 26 \\
3. & \multirow{2}{*}{ DVT } & 21 & Heparin & 9 & 40 \\
& & & LMWH & 5 & 22.7 \\
& & & Warfarin & 4 & 18.1 \\
& & & Acenocoumarol & 7 & 31.8 \\
4. & \multirow{2}{*}{ Others } & 14 & Heparin & 5 & 38.4 \\
& & & LMWH & 3 & 23 \\
& & & Warfarin & 2 & 15.3 \\
& & & Acenocoumarol & 4 & 30.7 \\
\hline
\end{tabular}

$\mathrm{N}$ : Number of patients, \%: Percentage

Table 3: Calculation of PDD for the anticoagulants

\begin{tabular}{llll}
\hline S. No. & Drug & Indication & PDD \\
\hline 1. & Warfarin (mg) & CAD & 4.00 \\
& & CVA & 3.32 \\
& DVT & 4.00 \\
2. & Heparin (U) & Others & 1.41 \\
& & CAD & 18731.71 \\
& CVA & 20000 \\
3. & DVT & 22222.22 \\
& Acenocoumarol (mg) & Others & 13700 \\
& CAD & 1.85 \\
& CVD & 1.16 \\
4. & DVT & 2.00 \\
& & Others & 2.00 \\
& LMWH (U) & CAD & 57.50 \\
& & CVD & 57.33 \\
& & DVT & 44.00 \\
& & Others & 80.00 \\
\hline
\end{tabular}

PDD: Prescribed daily dose, CAD: Coronary artery disease, CVA: Cerebrovascular accident, DVT: Deep vein thrombosis, LMWH: Low-molecular-weight heparin 
PDD was not the same as DDD, warfarin's dose was reduced to $10 \%$ than DDD, heparin was given in double doses than DDD, acenocoumarol was reduced to $20 \%$ than DDD, and LMWH was doubled than DDD and given. Although the dose varied on account of diagnosis, there was a marked difference in the dosing quantity (Table 4).

The comparison was carried out on a SAS system using simple t-test. The ratio between PDD and DDD of heparin for CAD, CVD, DVT, and others was $1.87,2,2.22$, and 1.37, respectively, the average PDD: DDD for heparin was 1.86. The PDD: DDD of LMWH for CAD, CVD, DVT, and others was $2.87,2.86,2.2$, and 4, respectively, and average ratio of PDD and DDD for LMWH was 2.9. Warfarin's PDD: DDD for CAD, CVD, DVT, and others was $0.53,0.44,0.53$, and 0.40 , respectively, and average ratio was found to be 0.46 , and the ratio of PDD and DDD of Acenocoumarol for CAD, CVD, DVT, and others was $0.37,0.23,0.4$, and 0.4 respectively, and the average PDD: DDD across all diseases for which acenocoumarol was used was found to be 0.75 . Heparin was greatly given in double doses and had $p<0.0001$ showing that there is a statistically significant difference, likewise, LMWH was also given in double doses having $\mathrm{p}<0.0001$, i.e., statistically significant difference in dosing. Whereas, warfarin was given in half of the defined doses which was also statistically significant considering its $p$ value to be $<0.0001$, likewise, acenocoumarol was even given in low doses even lower than half of the defined dose, showing $\mathrm{p}<0.0001$ it was also found to be statistically significant.

\section{DISCUSSION}

A total of 1500 cases were reviewed, out of which 119 cases were enrolled into the study according to the inclusion criteria, the remaining 1381 (92\%) cases were excluded based on the exclusion criteria, $730(48.6 \%)$ cases were excluded because there was no anticoagulant prescribed, $320(21 \%)$ patients hesitated to share their medication-related information, $186(12.4 \%)$ patients were receiving anticoagulants on hemodialysis, $121(8 \%)$ patients were suffering from end-stage renal disease, $15(1 \%)$ patients were diagnosed with cancer, and $9(0.6 \%)$ patients were suffering from psychiatric illness.

In this study and another study conducted by Klein et al. and recently Mosca et al. (2011) claimed that CAD was found to be predominant in men compared to women, accounting $60.5 \%$ were male and $39.5 \%$ were female, there was a slight increase of anticoagulant use in males compared to females $[9,10]$. In our study, $57.98 \%$ of the patients came from urban areas while $42.02 \%$ came from rural areas, with $p$ value of
0.92 which is $>0.05$, showing non-significance implying that patients are evenly distributed through urban and rural areas. Patients were seen majorly from the age groups belonging to 54-61, followed by $30-37$ and 46-53, followed by 38-45. In this study, majority of the cases $51.26 \%$ were confirmed with CAD were receiving anticoagulant medications. The study helped to identify the various anticoagulants which were being prescribed at the study site, out of which heparin was the most commonly preferred anticoagulants. Preferred anticoagulant in CAD has being heparin with 41 out of 61 patients receiving it [11]. Anticoagulants were also used in other cardiac diseases such as CRHD and AF. From our study, heparin or LMWH are first-line agents for DVT followed by oral anticoagulants.

We calculated PDD for the anticoagulants and were compared individually with their respective DDD defined by the WHO. We found that warfarin has being prescribed with half of its DDD at the study site $(\mathrm{p}<0.0001)$. Heparin was prescribed at almost double the DDD at the study site $(\mathrm{p}<0.0001)$. Acenocoumarol was given at slightly lesser doses than its DDD at the study site $(\mathrm{p}<0.0001)$. LMWH was given 3 times more than the DDD at the study site $(\mathrm{p}<0.0001)$. We have found that $79.82 \%$ of patients did not take on any dietary changes after initiation of anticoagulant therapy which is necessary in anticoagulation therapy, as stated by Klein et al. [9]. There were eight ADRs reported, out of which seven occurred in female and only one occurred in male. The reported ADRs were with acenocoumarol (5) and warfarin (3).

\section{CONCLUSION}

We observed that the Vitamin $\mathrm{K}$ antagonists were prescribed at half of the WHO defined doses. Heparin derivatives were prescribed at increased doses and sometimes double the WHO defined doses. We observed that the anticoagulants were not prescribed as the WHO defined doses in the study site. The prevalence of ADRs was found to be higher in acenocoumarol than warfarin.

\section{Study limitation}

The study was conducted in a single hospital in a limited period of time. Patients were not followed up after being shifted into cardiology department.

\section{CONFLICTS OF INTEREST}

No conflicts of interest were raised by the authors.

Table 4: Comparison of PDD:DDD

\begin{tabular}{|c|c|c|c|c|c|c|}
\hline S. No. & Drug & Indication & PDD & DDD & PDD:DDD & $p$ value \\
\hline \multirow[t]{5}{*}{1.} & HEPARIN & CAD & 18731.71 & 10000 & 1.87 & $<0.0001$ \\
\hline & & CVD & 20000 & & 2 & \\
\hline & & DVT & 22222.22 & & 2.22 & \\
\hline & & Others & 13700 & & 1.37 & \\
\hline & Overall & & 18663 & & 1.86 & \\
\hline \multirow[t]{5}{*}{2.} & LMWH & CAD & 57.5 & 20 & 2.87 & $<0.0001$ \\
\hline & & CVD & 57.3 & & 2.86 & \\
\hline & & DVT & 44.00 & & 2.2 & \\
\hline & & Others & 80.00 & & 4 & \\
\hline & Overall & & 59.7 & & 2.9 & \\
\hline \multirow[t]{5}{*}{3.} & WARFARIN & CAD & 4.00 & 7.5 & 0.53 & $<0.0001$ \\
\hline & & CVD & 3.32 & & 0.44 & \\
\hline & & DVT & 4.00 & & 0.53 & \\
\hline & & Others & 3.00 & & 0.40 & \\
\hline & Overall & & 3.50 & & 0.46 & \\
\hline \multirow[t]{5}{*}{4.} & ACENOCOUMAROL & CAD & 1.85 & 5 & 0.37 & $<0.0001$ \\
\hline & & CVD & 1.16 & & 0.23 & \\
\hline & & DVT & 2.00 & & 0.4 & \\
\hline & & Others & 2.00 & & 0.4 & \\
\hline & Overall & & 1.75 & & 0.75 & \\
\hline
\end{tabular}

PDD: Prescribed daily dose, DDD: Defined daily dose, CAD: Coronary artery disease, CVD: Cerebrovascular accident, DVT: Deep vein thrombosis, LMWH: Low-molecular-weight heparin 


\section{REFERENCES}

1. Parthasarthy G, Nyfort-Hansen K, Nahata M. Drugutilizayionevaluation. Sathvik B.S.A Textbook of Clinical Pharmacy Practice. Hyderabad: Universities Press; 2011. p. 447-59.

2. WHO. Introduction to Drug Utilization Research. Available from: https://www.whocc.no/filearchive/publications/drug utilization research.pdf. [Last accessed on 2016 Sep 10].

3. Mukesh K, Vicky D, Shruti M, Dinesh S, Neha M, Lahkar M. Cardiovascular disease prevalence and drug utilization patterns at a tertiary care hospital in Northeastern India. Int $\mathrm{J}$ Pharm Pharm Sci 2016;8:116-9.

4. Colledge NR, Walker BR, Ralston SH. Anticoagulant and Antithrombotic Therapy, Principles and Practice of Davidson's Medicine. $21^{\text {st }}$ ed. India: Elsevier Publications; 2012. p. 1014

5. Harvey RA. Anticoagulants, Lippincott's Illustrated Reviews, Pharmacology. $5^{\text {th }}$ ed. Philadelphia, PA: Wolters Liuwer Publications; 2011. p. 251-7.

6. Shazia A, Syed BS, Maqsood A. Drug utilization and economic impact of anticoagulants in unstable angina/non-ST elevation myocardial infarction in Karachi. Int J Pharm Pharm Sci 2015;7:183-5.

7. Galatro KM, Adams PC, Cohen M, McBride R, Blanke H. Bleeding complications and INR control of combined warfarin and low-dose aspirin therapy in patients with unstable angina and non-Q-wave myocardial infarction. J Thromb Thrombolysis 1998;5:249-55.

8. Di Biase L, Burkhardt JD, Santangeli P, Mohanty P, Sanchez JE, Horton R, et al. Periprocedural stroke and bleeding complications in patients undergoing catheter ablation of atrial fibrillation with different anticoagulation management: Results from the role of coumadin in preventing thromboembolism in atrial fibrillation (AF) patients undergoing catheter ablation (COMPARE) randomized trial. Circulation 2014;129:2638-44.

9. Klein W, Buchwald A, Hillis SE, Monrad S, Sanz G, Turpie AG, et al. Comparison of low-molecular-weight heparin with unfractionated heparin acutely and with placebo for 6 weeks in the management of unstable coronary artery disease. Fragmin in unstable coronary artery disease study (FRIC) Circulation 1997;96:61-8

10. Mosca L, Appel LJ, Benjamin EJ, Berra K, Chandra-Strobos N, Fabunmi RP, et al. Evidence-based guidelines for cardiovascular disease prevention in women. American heart association scientific statement. Arterioscler Thromb Vasc Biol 2004;24:e29-50.

11. Falck-Ytter Y, Francis CW, Johanson NA, Curley C, Dahl OE, Schulman S, et al. Prevention of VTE in orthopedic surgery patients: Antithrombotic therapy and prevention of thrombosis. $9^{\text {th }}$ ed. American college of chest physicians evidence-based clinical practice guidelines. Chest 2012;141:e278S-325. 